\title{
HALF-SPACE TYPE THEOREM FOR TRANSLATING SOLITONS OF THE MEAN CURVATURE FLOW IN EUCLIDEAN SPACE
}

\author{
DAEHWAN KIM AND JUNCHEOL PYO \\ (Communicated by Guofang Wei)
}

\begin{abstract}
In this paper, we determine which half-space contains a complete translating soliton of the mean curvature flow and it is related to the wellknown half-space theorem for minimal surfaces. We prove that a complete translating soliton does not exist with respect to the velocity $\mathrm{v}$ in a closed half-space $\mathcal{H}_{\widetilde{\mathrm{v}}}=\left\{x \in \mathbb{R}^{n+1} \mid\langle x, \widetilde{\mathrm{v}}\rangle \leq 0\right\}$ for $\langle\mathrm{v}, \widetilde{\mathrm{v}}\rangle>0$, whereas in a half-space $\mathcal{H}_{\widetilde{\mathrm{v}}},\langle\mathrm{v}, \widetilde{\mathrm{v}}\rangle \leq 0$, a complete translating soliton can be found. In addition, we extend this property to cones: there are no complete translating solitons with respect to v in a right circular cone $C_{\mathrm{v}, a}=\left\{x \in \mathbb{R}^{n+1} \mid\left\langle\frac{x}{\|x\|}, \mathrm{v}\right\rangle \leq a<1\right\}$.
\end{abstract}

\section{INTRODUCTION}

In minimal surface theory, the half-space theorem proved by Hoffman and Meeks [18] is a well-known global property: A connected, proper, possibly branched, nonplanar minimal surface in $\mathbb{R}^{3}$ is not contained in a half-space. They used two main elements, namely, the scaling invariance of minimality of the minimal surfaces in Euclidean space and the maximum principle. Specifically, the facts that a halfcatenoid converges to the punctured plane through a scale-down and the maximum principle were used to prove the half-space theorem. On the one hand, the generalization of the half-space theorem fails in a higher dimensional Euclidean space. In this case, a higher dimensional catenoid is contained in a domain bounded by two parallel hyperplanes. On the other hand, generalizations of the half-space theorem to other geometric surfaces, such as constant mean curvature surfaces, in various ambient spaces has been successful (see, [3, 10, 12, 28, 30, 33, 39]).

In this paper, we consider translating solitons of the mean curvature flow (MCF). A smooth family of immersions $F: \Sigma \times[0, T) \rightarrow \mathbb{R}^{n+1}$ is a solution of the MCF if $F$ satisfies the following parabolic equation:

$$
\frac{\partial}{\partial t} F(p, t)=\vec{H}(p, t)
$$

for all $(p, t) \in \Sigma \times[0, T)$, where $\vec{H}$ is the mean curvature vector. The MCF is a negative gradient flow of the area functional. It is also generally known that any closed hypersurface develops singularities within a finite time under the MCF. Therefore,

Received by the editors June 24, 2020, and, in revised form, November 12, 2020.

2020 Mathematics Subject Classification. Primary 53C42; Secondary 53E10.

The first author was supported by the National Research Foundation of Korea (NRF) grant funded by the Korea government (MSIT) (NRF-2019R1C1C1004819) and a KIAS Individual Grant (MG070801) at Korea Institute for Advanced Study.

The second author was supported by NRF (NRF-2017R1E1A1A03070495 and NRF2020R1A2C1A01005698). 
it is important to study the singularities of the MCF. Huisken [19] and Huisken and Sinestrari 20. demonstrated that there are two types of singularities, type-I and type-II, represented by a self-shrinker and a translating soliton, respectively. The translating soliton of the MCF is not only a blow-up limit flow of a type-II singularity but also a special solution that moves only in a constant direction $\mathrm{v}$ without deforming its shape under the MCF; the solution form is as follows:

$$
F(p, t)=F(p)+\mathrm{v} t
$$

where $F(p)=F(0, p)$.

For a self-shrinker, Cavalcante and Espinar 4] proved that the only properly immersed self-shrinker $\Sigma$ is contained in one of the closed half-spaces determined by a hyperplane $P$ is $\Sigma=P$. Some of the known translating solitons such as grim reaper cylinders, translating bowls and $\Delta$-wings are contained in a half-space. In [40], Shahriyari proved the non-existence of complete graphical translating solitons in a cylindrical domain and Møller 31 extended it to both a higher dimensional case and complete proper embedded translating solitons. Recently, Chini and Møller 8. demonstrated that there are no properly immersed $n$-dimensional translating solitons contained in the intersection domain of two transverse half-spaces that are parallel to $\mathrm{v}$ in $\mathbb{R}^{n+1}$. According to the various examples in Section 2 , there are complete translating solitons in a half-space that is containing v. However, such translating solitons are not contained in a half-space that does not contain v. This is generally true, as proved through the following theorem:

Theorem 1.1. There are no complete translating solitons for the velocity $\mathrm{v}$ under the MCF in a closed half-space $\mathcal{H}_{\widetilde{\mathrm{v}}}=\left\{x \in \mathbb{R}^{n+1} \mid\langle x, \widetilde{\mathrm{v}}\rangle \leq 0\right\}$ with $\langle\mathrm{v}, \widetilde{\mathrm{v}}\rangle>0$.

Corollary 1.2. There are no complete translating solitons in any bounded domain of $\mathbb{R}^{n+1}$.

Because translating solitons are minimal hypersurfaces in Euclidean space endowed with a conformally flat metric, they share an analogical property with minimal surfaces in Euclidean space. However, the Corollary 1.2 shows that translating solitons have a different property compared to that in the minimal surface theory. More precisely, a complete minimal surface in a ball in $\mathbb{R}^{3}$ exists, which was proved by Nadirashvili [32. We note that Chini and Møller [8] proved the non-existence of complete proper translating solitons in a bounded domain.

As another result in the minimal surface theory, Omori 37 considered the nonexistence of a minimal isometric immersion with the bounded below sectional curvature in the cones of Euclidean space using the existence of a sequence of points, which is now popularly known as the Omori-Yau maximum principle. The OmoriYau maximum principle was introduced in [6, 37, 42, and can be applied to noncompact cases: Omori [37] proved the Omori-Yau maximum principle for the Hessian of a function bounded from above on a complete Riemannian manifold with the sectional curvature bounded from below. Yau [42 and Cheng and Yau 6] established for the Laplacian of a function bounded from above on a complete Riemannian manifold with the Ricci curvature bounded from below. Various versions of the Omori-Yau maximum principle have been used to prove the geometric problems in [5, 7, 8, 25, 43]. A detailed introduction to various Omori-Yau maximum principles and their applications can be found in [1]. Based on the Omori-Yau maximum principle, we extend the results of Omori 37 for minimal surfaces to translating solitons of the MCF: 
Theorem 1.3. There are no complete translating solitons for the velocity $\mathrm{v}$ under the $M C F$ in a right circular cone $C_{\mathrm{v}, a}=\left\{x \in \mathbb{R}^{n+1} \mid\left\langle\frac{x}{\|x\|}, \mathrm{v}\right\rangle \leq a<1\right\}$.

As previous study, Impera and Rimoldi 21] demonstrated that an $n$-dimensional $f$-stochastically complete translating soliton cannot be contained in a lower cone containing the direction of the translation under the MCF. In [41, Xin established a version of the Omori-Yau maximum principle for complete proper translating solitons in Euclidean space, which is valid for a higher codimensional case.

\section{Translating solitons of the MCF}

Definition 2.1. A translating soliton $M$ with the velocity $\mathrm{v}$ is a hypersurface in $\mathbb{R}^{n+1}$ if it is a solution to the MCF such that the solution form is as follows:

$$
F(p, t)=F(p)+\mathrm{v} t,
$$

where $F(p)=F(p, 0)$. As an equivalent condition, it satisfies the following equation:

$$
\vec{H}=\mathrm{v}^{\perp}
$$

where $\vec{H}$ is the mean curvature vector field of $M$.

The velocity $\mathrm{v}$ indicates the direction of the translation of the translating soliton under the MCF. Up to scaling and rotation in $\mathbb{R}^{n+1}$, it is possible to assume $\mathrm{v}=$ $e_{n+1}$. There are various translating solitons, some of which are listed herein. We can find more translating solitons for examples, see [8, 15, 27] and the references therein.

Example 2.2 (Product of minimal submanifold). The simplest translating soliton is a plane parallel to $\mathrm{v}$ in $\mathbb{R}^{3}$ as a product of a line and $\mathbb{R}$ parallel to the direction $\mathrm{v}$. From this perspective, the translating solitons in $\mathbb{R}^{n+1}$ can be constructed as the product of an $(n-1)$-dimensional minimal submanifold $M$ and $\mathbb{R}$ parallel to the direction $\mathrm{v}$, i.e., $M \times \mathbb{R}$. There are numerous of translating solitons arising from minimal submanifolds. In 32, Nadirashvili constructed a complete, non-proper, minimal disk in the unit ball. Specifically, a complete non-proper translating soliton can be obtained from Nadirashvili's minimal surface.

Example 2.3 (Grim reaper cylinders). The grim reaper $y=-\log \cos (x)$ is a translating soliton on $\mathbb{R}^{2}$, i.e., the only eternal solution of the MCF in $\mathbb{R}^{2}$, which is also known as the curve-shortening flow. Its product surface, which is a cylindrical surface of the grim reaper, is called a canonical grim reaper cylinder whose suitable combination of rotation and dilation is called a grim reaper cylinder. The following parametrization is for a family of grim reaper cylinders:

$$
X_{\theta}(u, v)=\left(s, t,-\frac{1}{\cos ^{2}(\theta)} \log \cos (s \cos (\theta))+t \tan (\theta)\right) .
$$

In particular, the grim reaper cylinder is a one-parameter family of cylindrical surfaces from the canonical grim reaper cylinder to the plane parallel to $\mathrm{v}=e_{3}$.

Example 2.4 (Translating bowl and winglike translator). Altschuler and $\mathrm{Wu}[2]$ and Clutterbuck, Schnürer and Schulze [9] showed the existence of the translating bowl and the winglike translator. These are rotationally symmetric translating solitons and can thus be represented as an immersion $X: I \times \mathbb{S}^{n-1} \rightarrow \mathbb{R}^{n+1}$ parametrized by

$$
X\left(s, \phi_{1}, \cdots, \phi_{n-1}\right)=\left(x(s) \Phi\left(\phi_{1}, \cdots, \phi_{n-1}\right), y(s)\right),
$$


where $\Phi$ is an orthogonal parametrization of the $(n-1)$-dimensional unit sphere. The profile curve $\gamma(s)=(x(s), y(s))$ parametrized by arc-length satisfies the following differential equation:

$$
x^{\prime}(1-n)+n y y^{\prime}+y\left(x^{\prime} y^{\prime \prime}-x^{\prime \prime} y^{\prime}\right)=0 .
$$

In particular, the translating bowl and winglike translator have an asymptotic behavior of $y=x^{2}$. The authors 24] rediscovered their asymptotic behaviors of the profile curve using the phase-plane method to the above differential equation.

Example 2.5 (Generalized winglike translator). As a generalization of the winglike translator, Kunikawa [26] constructed an $m$-dimensional translating soliton in $\mathbb{R}^{n}$. Let $N$ be any minimal submanifold in $\mathbb{S}^{n-2} \subset \mathbb{R}^{n-1}$ and $r:[0, \infty] \rightarrow \mathbb{R}$ be a function satisfying

$$
r^{\prime \prime}=\left(1+r^{\prime 2}\right)\left(1-\frac{(m-1) r^{\prime}}{t}\right)
$$

which is an $m$-dimensional winglike translator equation. The immersion $F: M \rightarrow$ $\mathbb{R}^{n}$ defined by $F(t, p)=(t p, r(t))$ where $p \in N$ and $t \in[0, \infty]$ is an $m$-dimensional translating soliton with the velocity $e_{n} \in \mathbb{R}^{n}$.

Example 2.6 (Helicoidal translating solitons). Halldorsson [13] proved the existence of the helicoidal rotating solitons under the MCF, which are also known as the helicoidal translating solitons. The authors 24] completely classified the profile curves and analyzed their asymptotic behaviors in the same way as those of the translating bowl or winglike translator. Consider a helicoidal translating soliton $\Sigma$ with the pitch $h$ whose helicoidal axis is the $z$-axis. We can parametrize $\Sigma$ as $X: \Sigma \rightarrow \mathbb{R}^{3}$ by

$$
X(s, t)=(x(s) \cos (t), x(s) \sin (t), y(s)+h t),
$$

such that the profile curve $(x, y)$ parametrized by arc-length satisfies the following differential equation:

$$
\left(x^{2}+2 h^{2} x^{2}\right) y^{\prime}+x\left(h^{2}+x^{2}\right)\left(x^{\prime} y^{\prime \prime}-y^{\prime} x^{\prime \prime}\right)-2 x x^{\prime}\left(x^{2}+h^{2} x^{2}\right)=0 .
$$

Example 2.7 ( $\Delta$-wing). The existence of $\Delta$-wings, which are graphs over the strips $\mathbb{R} \times(-b, b)$ and where $b>\frac{\pi}{2}$, was proved by Hoffman, Ilmanen, Martín and White [14. In particular, $\Delta$-wing has the asymptotic behaviors of grim reaper cylinders with the angle $\theta= \pm \arccos (2 b / \pi)$. They [14] also proved the following classification theorem: For every $b>\frac{\pi}{2}$, there is (up to the translation) a unique, complete and strictly convex translating soliton $u: \mathbb{R} \times(-b, b) \rightarrow \mathbb{R}$, i.e., the only other complete translating graphs are the grim reaper cylinders and the translating bowl.

Example 2.8 (Semi-graph translating soliton). Hoffman, Martín and White [16] proved the existence and uniqueness of a two-parameter family of translating solitons of the MCF. The family has several types of translating solitons, namely, Scherkenoids, Scherk translators, pithchfork translators and helicoid-like translators. The constructions of graphical translating solitons are over parallelograms and strips with infinite boundary values, which are derived from the minimal surface theory because the translating soliton is a minimal surface in a metric measure space that is conformally changed from Euclidean metric. 
Example 2.9 (Translating solitons using gluing technique). Using the grim reaper cylinder and the plane as building blocks, Nguyen 34] constructed the new examples of self-translating solitons with four ends using a gluing technique, two of which are asymptotic to planes and the other two ends are exponentially asymptotic to a grim reaper cylinder, which are called the translating tridents. Specifically, Hoffman, Martín and White [17 rediscovered the existence of a one-parametric family of the translating solitons, i.e., Nguyen's tridents. Nguyen 36] also constructed doubly periodic translating solitons with numerous but finite grim reaper cylinders during each period, which is an extension of the result of Nguyen [35] on the desingularization of a finite family of grim reaper cylinders. Dávila, del Pino and Nguyen [11] constructed embedded, complete translating solitons homeomorphic to the Costa-Hoffman-Meeks minimal surfaces, which are a desingularization of the union of a winglike translator and a translating bowl.

The proofs of Theorems 1.1 and 1.3 are provided in Sections 3 and 4, respectively and are based on the following the Omori-Yau maximum principle:

Theorem 2.10 (Omori-Yau maximum principle). Let $M$ be a connected and complete Riemannian manifold with Ricci curvature bounded below. Then, there exists a sequence $\left\{x_{k}\right\}$ on $M$ such that

$$
\lim _{k \rightarrow \infty} f\left(x_{k}\right)=f^{*}, \quad\left\|\nabla f\left(x_{k}\right)\right\|<\frac{1}{k}, \quad \triangle f\left(x_{k}\right)<\frac{1}{k},
$$

for any $f \in C^{2}(M)$ with $f^{*}=\sup _{M} f<\infty$.

\section{Proof of Theorem 1.1}

Let $M$ be an $n$-dimensional complete translating soliton with the velocity v under the $\mathrm{MCF}$ in $\mathbb{R}^{n+1}$. For the unit normal vector $\widetilde{\mathrm{v}}$ of the closed half-space $\mathcal{H}_{\widetilde{\mathrm{v}}}$, we denote $c=\langle\mathrm{v}, \widetilde{\mathrm{v}}\rangle>0$. Let us assume that $M \subset \mathcal{H}_{\widetilde{\mathrm{v}}}=\left\{x \in \mathbb{R}^{n+1} \mid\langle x, \widetilde{\mathrm{v}}\rangle \leq 0\right\}$.

To prove that the Ricci curvature of $M$ is bounded below, we take an orthonormal basis $\left\{e_{k}\right\}_{k=1}^{n}$ of $T_{p} M$ in which the second fundamental form $A(p)$ is diagonal at the considered point $p$ on $M$. The Ricci curvature with respect to $\left\{e_{k}\right\}_{k=1}^{n}$ at $p$ is then

$$
\begin{aligned}
\operatorname{Ric}_{p}\left(e_{i}, e_{i}\right) & =-\sum_{j=1, j \neq i}^{n} \kappa_{j}(p) \kappa_{i}(p) \\
& =\kappa_{i}^{2}(p)-\sum_{j=1}^{n} \kappa_{j}(p) \kappa_{i}(p) \\
& =\kappa_{i}^{2}(p)-H(p) \kappa_{i}(p) .
\end{aligned}
$$

The following inequality holds from $-1 \leq H \leq 1$ and equation (3.1):

$$
\operatorname{Ric}_{p}\left(e_{i}, e_{i}\right)=-H(p) \kappa_{i}(p)+\kappa_{i}^{2}(p)=\left(\kappa_{i}(p)-\frac{H(p)}{2}\right)^{2}-\frac{H^{2}(p)}{4} \geq-\frac{1}{4} .
$$

Therefore, the Omori-Yau maximum principle holds on $M$.

We assume $\widetilde{\mathrm{v}}=\mathrm{v}+a$, where $a$ is a constant vector and $\widetilde{\mathrm{v}}$ and $\mathrm{v}$ are unit constant vectors such that $\langle\widetilde{\mathrm{v}}, \mathrm{v}\rangle=c$, where $c$ is a positive constant. We define the height 
function $\phi=\langle x, \widetilde{\mathrm{v}}\rangle$ of $M$ with respect to $\widetilde{\mathrm{v}}$. By direct computation, we obtain the following:

$$
\begin{aligned}
\|\nabla \phi\|^{2} & =\left\|\widetilde{\mathrm{v}}^{\top}\right\|^{2}=1-\left\|\widetilde{\mathrm{v}}^{\perp}\right\|^{2}, \\
\triangle \phi & =H\langle\nu, \widetilde{\mathrm{v}}\rangle .
\end{aligned}
$$

By the Omori-Yau maximum principle, there is a sequence $\left\{x_{k}\right\}$ on $M$ such that

$$
\begin{aligned}
\lim _{k \rightarrow \infty} \phi\left(x_{k}\right) & =\sup _{M} \phi<0, \\
\frac{1}{k^{2}} & >\left\|\nabla \phi\left(x_{k}\right)\right\|^{2}=1-\left\|\widetilde{\mathrm{v}}^{\perp}\left(x_{k}\right)\right\|^{2}, \\
\frac{1}{k} & >\triangle \phi\left(x_{k}\right)=H\left(x_{k}\right)\left\langle\nu\left(x_{k}\right), \widetilde{\mathrm{v}}\right\rangle .
\end{aligned}
$$

We observe the following from the equation (3.2):

$$
\lim _{k \rightarrow \infty}\left\|\widetilde{\mathrm{v}}^{\perp}\left(x_{k}\right)\right\|^{2}=1
$$

which indicates that $\nu$ becomes parallel to $\widetilde{\mathrm{V}}$ as $k$ tends toward infinity. By the inequality (3.3), we obtain the following equation after passing to the sequence $\left\{x_{k}\right\}$ :

$$
0 \geq \lim _{k \rightarrow \infty} \triangle \phi\left(x_{k}\right)=\lim _{k \rightarrow \infty}\left\langle\nu\left(x_{k}\right), \mathrm{v}\right\rangle\left\langle\nu\left(x_{k}\right), \widetilde{\mathrm{v}}\right\rangle=\langle\widetilde{\mathrm{v}}, \mathrm{v}\rangle=c>0
$$

Therefore, we arrive at a contradiction. As a consequence, there are no complete translating solitons in $\mathcal{H}_{\widetilde{\mathrm{v}}}$.

Remark 3.1. To prove Theorem 1.1, we use the condition $\langle\widetilde{\mathrm{v}}, \mathrm{v}\rangle=c>0$. If we consider $\langle\widetilde{\mathrm{v}}, \mathrm{v}\rangle \leq 0$, then there are several counter examples, namely, hyperplanes parallel to $\mathrm{v}$ if $\langle\widetilde{\mathrm{v}}, \mathrm{v}\rangle=0$ and grim reaper cylinders or translating bowls if $\langle\widetilde{\mathrm{v}}, \mathrm{v}\rangle\langle 0$. The novelty of Theorem 1.1 is twofold. Firstly, the theorem holds for any dimension. Secondly, for the half-space theorem for minimal surfaces in Euclidean space, the properness is a necessary condition. The theorem holds regardless of properness on the translating solitons.

Remark 3.2. Chini and Møller 8 proved a half-space type theorem of a proper translating soliton in a bi-half-space, which is the intersection domain of two transverse half-spaces that are parallel to $\mathrm{v}$ in $\mathbb{R}^{n+1}$. It directly follows that there are no proper translating solitons in any bounded domain of $\mathbb{R}^{n+1}$. However, as mentioned in Remark 3.1. Theorem 1.1 and Corollary 1.2 hold regardless of whether the translating soliton is proper or not.

\section{Proof of Theorem 1.3}

Let $M$ be an $n$-dimensional complete translating soliton with $\mathrm{v}$ in a right circular cone $C_{\mathrm{v}, a}=\left\{x \in \mathbb{R}^{n+1} \mid\left\langle\frac{x}{\|x\|}, \mathrm{v}\right\rangle \leq a<1\right\}$, where $a$ is a constant. For $C_{\mathrm{v}, a} \subset \mathcal{H}_{\mathrm{v}}$ with $a \leq 0$, it is sufficient to consider only $0<a<1$ based on Theorem 1.1. We already know that the Ricci curvature of $M$ is bounded from below by $-\frac{1}{4}$. Thus, the Omori-Yau maximum principle holds on $M$. 
By direct computation, we can calculate the following equations:

$$
\begin{aligned}
\nabla\|x\| & =\frac{x^{\top}}{\|x\|}, \\
\|\nabla\| x\|\|^{2} & =\frac{\left\|x^{\top}\right\|^{2}}{\|x\|^{2}} \leq 1 \\
\triangle\|x\| & =\frac{1}{\|x\|}\left(\frac{1}{2} \triangle\|x\|^{2}-\|\nabla\| x\|\|^{2}\right)=\frac{1}{\|x\|}\left(\langle x, \vec{H}\rangle+n-\|\nabla\| x\|\|^{2}\right) .
\end{aligned}
$$

We define the non-positive function $\psi=\langle x, \mathrm{v}\rangle-a\|x\|$ that satisfies the following:

$$
\begin{aligned}
\|\nabla \psi\| & =\left\|\mathrm{v}^{\top}-a \nabla\right\| x\|\|=\left\|\mathrm{v}^{\top}-a \frac{x^{\top}}{\|x\|}\right\| \geq\left|\left\|\mathrm{v}^{\top}\right\|-a \frac{\left\|x^{\top}\right\|}{\|x\|}\right|, \\
\Delta \psi & =H^{2}-a \triangle\|x\|,
\end{aligned}
$$

By applying the Omori-Yau maximum principle to $\psi$, there is a sequence $\left\{x_{k}\right\}$ such that

$$
\begin{aligned}
\lim _{k \rightarrow \infty} \psi\left(x_{k}\right) & =\sup _{M} \psi \leq 0, \\
\frac{1}{k} & >\left\|\nabla \psi\left(x_{k}\right)\right\|, \\
\frac{1}{k} & >\Delta \psi\left(x_{k}\right) .
\end{aligned}
$$

From the equation (4.1), we first consider the following:

$$
\left\|\mathrm{v}^{\top}\left(x_{k}\right)\right\|-\frac{1}{k}<a \frac{\left\|x_{k}^{\top}\right\|}{\left\|x_{k}\right\|}<\frac{1}{k}+\left\|\mathrm{v}^{\top}\left(x_{k}\right)\right\| .
$$

If $H\left(x_{k}\right)$ converges to zero as $k \rightarrow \infty$, then $\left\|\mathrm{v}^{\top}\left(x_{k}\right)\right\|=1$ and we have a direct contradiction. Because $H$ is bounded, we can consider a subsequence $\left\{x_{k_{l}}\right\}$ such that $H\left(x_{k_{l}}\right)$ converges as $l \rightarrow \infty$. Thus, the subsequence guarantees the property of the Omori-Yau maximum principle and the convergence of $H\left(x_{k_{l}}\right)$. We replace the sequence of the Omori-Yau maximum principle with the subsequence $\left\{x_{k_{l}}\right\}$, which indicates that both $\left\|\mathrm{v}^{\top}\left(x_{k_{l}}\right)\right\|^{2}$ and $\frac{\left\|x_{k_{l}}\right\|^{2}}{\left\|x_{k_{l}}\right\|^{2}}$ converge as $l \rightarrow \infty$. From the equation (4.3), we have the following equation:

$$
1-\lim _{l \rightarrow \infty} H^{2}\left(x_{k_{l}}\right)=a^{2}\left(1-\lim _{l \rightarrow \infty} \frac{\left\|x_{k_{l}}^{\perp}\right\|^{2}}{\left\|x_{k_{l}}\right\|^{2}}\right)
$$

Thus, by multiplying with $H^{2}\left(x_{k_{l}}\right)$, we obtain the following equation:

$$
\lim _{l \rightarrow \infty} a^{2}\left\langle\frac{x_{k_{l}}}{\left\|x_{k_{l}}\right\|}, \vec{H}\left(x_{k_{l}}\right)\right\rangle^{2}=\lim _{l \rightarrow \infty}\left(H^{4}\left(x_{k_{l}}\right)-\left(1-a^{2}\right) H^{2}\left(x_{k_{l}}\right)\right) .
$$

In particular, the following inequality is obtained:

$$
\lim _{l \rightarrow \infty}\left(H^{2}\left(x_{k_{l}}\right)+a^{2}-1\right) \geq 0 .
$$


From the equation (4.2), we then have

$$
\begin{aligned}
\frac{1}{k}>\Delta \psi\left(x_{k}\right) & =H^{2}\left(x_{k}\right)-a\left(\left\langle\frac{x_{k}}{\left\|x_{k}\right\|}, \vec{H}\left(x_{k}\right)\right\rangle+\frac{n}{\left\|x_{k}\right\|}-\frac{\|\nabla\| x_{k}\|\|^{2}}{\left\|x_{k}\right\|}\right) \\
& \geq H^{2}\left(x_{k}\right)-a\left|\left\langle\frac{x_{k}}{\left\|x_{k}\right\|}, \vec{H}\left(x_{k}\right)\right\rangle\right|-\frac{a n}{\left\|x_{k}\right\|} .
\end{aligned}
$$

Thus, the following inequality holds:

$$
\frac{1}{k}+\frac{a n}{\left\|x_{k}\right\|}>H^{2}\left(x_{k}\right)-a\left|\left\langle\frac{x_{k}}{\left\|x_{k}\right\|}, \vec{H}\left(x_{k}\right)\right\rangle\right| .
$$

We replace the sequence of the Omori-Yau maximum principle with the subsequence $\left\{x_{k_{l}}\right\}$. Inserting the equation (4.4) into the inequality (4.5) and passing to the subsequence $\left\{x_{k_{l}}\right\}$, the following inequality is obtained:

$$
\text { (4.6) } \lim _{l \rightarrow \infty}\left(\frac{1}{k_{l}}+\frac{a n}{\left\|x_{k_{l}}\right\|}\right) \geq \lim _{l \rightarrow \infty}\left(H^{2}\left(x_{k_{l}}\right)-\sqrt{H^{4}\left(x_{k_{l}}\right)-\left(1-a^{2}\right) H^{2}\left(x_{k_{l}}\right)}\right) \text {. }
$$

In addition, we define a positive function defined on $\left[1-a^{2}, 1\right]$ as

$$
\alpha(t)=t-\sqrt{t^{2}-\left(1-a^{2}\right) t} .
$$

Bacause $\alpha^{\prime}(t)<0, \alpha(t)$ is a decreasing and positive function such that $\alpha(1)=1-a$ is the minimum value in $\left[1-a^{2}, 1\right]$. It is possible to assume $\inf _{M}\|x\| \geq \frac{2 a n}{1-a}$ through a translation along the direction $-\mathrm{v}$. We then arrive at a contradiction using the inequality (4.6) as follows:

$$
\begin{aligned}
0 & \geq \lim _{l \rightarrow \infty} \alpha\left(H^{2}\left(x_{k_{l}}\right)\right)-\lim _{l \rightarrow \infty}\left(\frac{1}{k_{l}}+\frac{a n}{\left\|x_{k_{l}}\right\|}\right) \\
& \geq 1-a-\lim _{l \rightarrow \infty}\left(\frac{1}{k_{l}}+\frac{1-a}{2}\right) \\
& \geq \frac{1-a}{2}>0 .
\end{aligned}
$$

Therefore, there are no complete translating solitons with the direction $\mathrm{v}$ of the translation under the $\mathrm{MCF}$ in $\mathcal{C}_{\mathrm{v}, a} \subset \mathbb{R}^{n+1}$.

Remark 4.1. Because we consider $-1 \leq a<1$ in Theorem 1.3. Theorem 1.1 is induced by Theorem 1.3. There are several counter examples of complete translating solitons if the right circular cone contains $\mathrm{v}$, namely, the translating bowls and winglike translators are contained in $\left\{x \in \mathbb{R}^{n+1} \mid\left\langle\frac{x}{\|x\|}, \mathrm{v}\right\rangle \geq a\right\}$ for a constant $a$.

\section{REFERENCES}

[1] Luis J. Alías, Paolo Mastrolia, and Marco Rigoli, Maximum principles and geometric applications, Springer Monographs in Mathematics, Springer, Cham, 2016. MR3445380

[2] Steven J. Altschuler and Lang F. Wu, Translating surfaces of the non-parametric mean curvature flow with prescribed contact angle, Calc. Var. Partial Differential Equations 2 (1994), no. 1, 101-111, DOI 10.1007/BF01234317. MR1384396

[3] Marcos Petrúcio Cavalcante and Wagner Oliveira Costa-Filho, The halfspace theorem for minimal hypersurfaces in regions bounded by minimal cones, Bull. Lond. Math. Soc. 51 (2019), no. 4, 639-644, DOI 10.1112/blms.12265. MR3990382

[4] Marcos P. Cavalcante and José M. Espinar, Halfspace type theorems for self-shrinkers, Bull. Lond. Math. Soc. 48 (2016), no. 2, 242-250, DOI 10.1112/blms/bdv099. MR3483061

[5] Qun Chen and Hongbing Qiu, Rigidity of self-shrinkers and translating solitons of mean curvature flows, Adv. Math. 294 (2016), 517-531, DOI 10.1016/j.aim.2016.03.004. MR3479571 
[6] S. Y. Cheng and S. T. Yau, Differential equations on Riemannian manifolds and their geometric applications, Comm. Pure Appl. Math. 28 (1975), no. 3, 333-354, DOI 10.1002/cpa.3160280303. MR.385749

[7] Shiu Yuen Cheng and Shing Tung Yau, Maximal space-like hypersurfaces in the LorentzMinkowski spaces, Ann. of Math. (2) 104 (1976), no. 3, 407-419, DOI 10.2307/1970963. MR 431061

[8] F. Chini and N. M. Møller, Bi-halfspace and convex hull theorems for translating solitons, Int. Math. Res. Not., rnz183, https://doi.org/10.1093/imrn/rnz183.

[9] Julie Clutterbuck, Oliver C. Schnürer, and Felix Schulze, Stability of translating solutions to mean curvature flow, Calc. Var. Partial Differential Equations 29 (2007), no. 3, 281-293, DOI 10.1007/s00526-006-0033-1. MR2321890

[10] Benoît Daniel, William H. Meeks III, and Harold Rosenberg, Half-space theorems for minimal surfaces in $\mathrm{Nil}_{3}$ and $\mathrm{Sol}_{3}$, J. Differential Geom. 88 (2011), no. 1, 41-59. MR2819755

[11] Juan Dávila, Manuel del Pino, and Xuan Hien Nguyen, Finite topology self-translating surfaces for the mean curvature flow in $\mathbb{R}^{3}$, Adv. Math. 320 (2017), 674-729, DOI 10.1016/j.aim.2017.09.014. MR.3709119

[12] Henrique F. de Lima and Márcio S. Santos, Height estimates and half-space type theorems in weighted product spaces with nonnegative Bakry-Émery-Ricci curvature, Ann. Univ. Ferrara Sez. VII Sci. Mat. 63 (2017), no. 2, 323-332, DOI 10.1007/s11565-016-0268-5. MR3712444

[13] Hoeskuldur P. Halldorsson, Helicoidal surfaces rotating/translating under the mean curvature flow, Geom. Dedicata 162 (2013), 45-65, DOI 10.1007/s10711-012-9716-2. MR3009534

[14] D. Hoffman, T. Ilmanen, F. Martín, and B. White, Graphical translators for mean curvature flow, Calc. Var. Partial Differential Equations 58 (2019), no. 4, Paper No. 117, 29, DOI 10.1007/s00526-019-1560-x. MR3962912

[15] D. Hoffman, T. Ilmanen, F. Martín, and B. White, Correction to: Graphical translators for mean curvature flow, Calc. Var. Partial Differential Equations 58 (2019), no. 4, Paper No. 158, 1, DOI 10.1007/s00526-019-1601-5. MR4029723

[16] D. Hoffman, F. Martín, and B. White, Scherk-like translators for mean curvature flow, to appear in J. Differential Geom. arXiv:1903.04617.

[17] D. Hoffman, F. Martín, and B. White, Nguyen's tridents and the classification of semigraphical translators for mean curvature flow, arXiv:1909.09241.

[18] D. Hoffman and W. H. Meeks III, The strong halfspace theorem for minimal surfaces, Invent. Math. 101 (1990), no. 2, 373-377, DOI 10.1007/BF01231506. MR.1062966

[19] Gerhard Huisken, Asymptotic behavior for singularities of the mean curvature flow, J. Differential Geom. 31 (1990), no. 1, 285-299. MR1030675

[20] Gerhard Huisken and Carlo Sinestrari, Mean curvature flow singularities for mean convex surfaces, Calc. Var. Partial Differential Equations 8 (1999), no. 1, 1-14, DOI 10.1007/s005260050113. MR.1666878

[21] Debora Impera and Michele Rimoldi, Rigidity results and topology at infinity of translating solitons of the mean curvature flow, Commun. Contemp. Math. 19 (2017), no. 6, 1750002, 21, DOI 10.1142/S021919971750002X. MR3691501

[22] Debora Impera and Michele Rimoldi, Quantitative index bounds for translators via topology, Math. Z. 292 (2019), no. 1-2, 513-527, DOI 10.1007/s00209-019-02276-y. MR 3968913

[23] Daehwan Kim and Juncheol Pyo, Translating solitons foliated by spheres, Internat. J. Math. 28 (2017), no. 1, 1750006, 11, DOI 10.1142/S0129167X17500069. MR3611054

[24] Daehwan Kim and Juncheol Pyo, Existence and asymptotic behavior of helicoidal translating solitons of the mean curvature flow, Discrete Contin. Dyn. Syst. 38 (2018), no. 11, 5897-5919, DOI 10.3934/dcds.2018256. MR3917792

[25] Daehwan Kim and Juncheol Pyo, $O(m) \times O(n)$-invariant homothetic solitons for inverse mean curvature flow in $\mathbb{R}^{m+n}$, Nonlinearity 32 (2019), no. 10, 3873-3911, DOI 10.1088/13616544/ab272b. MR 4002403

[26] Keita Kunikawa, Translating solitons in arbitrary codimension, Asian J. Math. 21 (2017), no. 5, 855-872, DOI 10.4310/AJM.2017.v21.n5.a4. MR.3767268

[27] Francisco Martín, Andreas Savas-Halilaj, and Knut Smoczyk, On the topology of translating solitons of the mean curvature flow, Calc. Var. Partial Differential Equations 54 (2015), no. 3, 2853-2882, DOI 10.1007/s00526-015-0886-2. MR3412395

[28] Laurent Mazet, A general halfspace theorem for constant mean curvature surfaces, Amer. J. Math. 135 (2013), no. 3, 801-834, DOI 10.1353/ajm.2013.0027. MR3068403 
[29] L. Mazet and G. A. Wanderley, A half-space theorem for graphs of constant mean curvature $0<H<\frac{1}{2}$ in $\mathbb{H}^{2} \times \mathbb{R}$, Illinois J. Math. 59 (2015), no. 1, 43-53. MR3459627

[30] Heudson Mirandola, Half-space type theorems in warped product spaces with one-dimensional factor, Geom. Dedicata 138 (2009), 117-127, DOI 10.1007/s10711-008-9302-9. MR2469991

[31] N. M. Møller, Non-existence for self-translating solitons, arXiv:1411.2319

[32] Nikolai Nadirashvili, Hadamard's and Calabi-Yau's conjectures on negatively curved and minimal surfaces, Invent. Math. 126 (1996), no. 3, 457-465, DOI 10.1007/s002220050106. MR.1419004

[33] Barbara Nelli and Ricardo Sa Earp, A halfspace theorem for mean curvature $H=\frac{1}{2}$ surfaces in $\mathbb{H}^{2} \times \mathbb{R}$, J. Math. Anal. Appl. 365 (2010), no. 1, 167-170, DOI 10.1016/j.jmaa.2009.10.031. $\operatorname{MR} 2585087$

[34] Xuan Hien Nguyen, Translating tridents, Comm. Partial Differential Equations 34 (2009), no. 1-3, 257-280, DOI 10.1080/03605300902768685. MR2512861

[35] Xuan Hien Nguyen, Complete embedded self-translating surfaces under mean curvature flow, J. Geom. Anal. 23 (2013), no. 3, 1379-1426, DOI 10.1007/s12220-011-9292-y. MR3078359

[36] Xuan Hien Nguyen, Doubly periodic self-translating surfaces for the mean curvature flow, Geom. Dedicata 174 (2015), 177-185, DOI 10.1007/s10711-014-0011-2. MR.3303047

[37] Hideki Omori, Isometric immersions of Riemannian manifolds, J. Math. Soc. Japan 19 (1967), 205-214, DOI 10.2969/jmsj/01920205. MR215259

[38] Juncheol Pyo, Compact translating solitons with non-empty planar boundary, Differential Geom. Appl. 47 (2016), 79-85, DOI 10.1016/j.difgeo.2016.03.003. MR3504920

[39] Lucio Rodriguez and Harold Rosenberg, Half-space theorems for mean curvature one surfaces in hyperbolic space, Proc. Amer. Math. Soc. 126 (1998), no. 9, 2755-2762, DOI 10.1090/S0002-9939-98-04510-9. MR.1458259

[40] Leili Shahriyari, Translating graphs by mean curvature flow, Geom. Dedicata 175 (2015), 57-64, DOI 10.1007/s10711-014-0028-6. MR3323629

[41] Y. L. Xin, Translating solitons of the mean curvature flow, Calc. Var. Partial Differential Equations 54 (2015), no. 2, 1995-2016, DOI 10.1007/s00526-015-0853-y. MR3396441

[42] Shing Tung Yau, Harmonic functions on complete Riemannian manifolds, Comm. Pure Appl. Math. 28 (1975), 201-228, DOI 10.1002/cpa.3160280203. MR431040

[43] Shing Tung Yau, A general Schwarz lemma for Kähler manifolds, Amer. J. Math. 100 (1978), no. 1, 197-203, DOI 10.2307/2373880. MR 486659

Department of Mathematics Education, Daegu University, Gyeongsan-si, GyeongsangBUK-DO, 38453, RePUblic OF KorEA

Email address: daehwan@daegu.ac.kr

Department of Mathematics, Pusan National University, Busan 46241, Republic of Korea; and School of Mathematics, Korea Institute for Advanced Study, Seoul 02455, REPUBLIC OF KoREA

Email address: jcpyo@pusan.ac.kr 